\title{
Addendum to the paper \\ ON THE INVERSION OF THE CAUER-ROUTH MATRIX
}

\author{
BY \\ W. H. INGRAM \\ Quarterly of A pplied Mathematics XXVII, 215-222
}

This note extends the paper to networks with conductively separate but inductively linked parts. The practical problem of power transmission from energy sources in local networks of this kind to energy sinks in other local networks of the same kind via tielines is best solved by inversion of the Cauer-Routh matrix discussed in the main portion of the paper, exactly as in the case of a connected nonseparable network if by [ח, $K$ ] we understand the Veblen-Poincaré incidence matrix $[\bar{\Pi}, \bar{K}]$ with all linearly dependent rows deleted. But a question arises as to the propriety of denoting vertices corresponding to the deleted rows as ground-points when the local networks are far apart. Groundpoints in physics are points in the surface of a poorly conducting half-space and there are no handbook formulas for the resistance and other properties of the earth between separated ground-points. Because of its physical properties, the earth is unsuitable for power transmission and because of this and other reasons, tie-lines are always multiwire-almost always 3-wire.

The graph is a perfectly valid representation of the physical network (1) when we define a local netucork to be one where the ground-points are effectively the same point as when a heavy wire of very low specific resistance is taken for their location and (2) when the system consists of local networks and multi-wire tic-lines connecting them. In both cases an actual connection to earth of the ground-points can have no effect on the problem of calculating the currents which flow in response to sinusoidal isoperiodic e.m.f.s in the branches of the network.

Box 353, Fire Island

New York 11770 